\title{
La inclusión de la computadora en el aula por docentes de quinto grado de básica primaria como herramienta para propiciar el aprendizaje significativo en los estudiantes
}

FECHA DE RECEPCIÓN: 23 de junio FECHA DE APROBACIÓN: 17 de julio Pp. 50-63
Nancy Yamile Monsalve Castro* Carolina Monsalve Castro**

Incurporation of the Computer by

Teachers in Fifth Grade

Classruoms of Primary

Education as a Tod to Facilitate

Students' Meaningful Learning

L'intruduction de l'ardinateur en

classe comme outil de formation

et de promotion d'un

apprentissage significatif

chez les élèves de cinquième

année d'écule élémentaire

A inc/usão da computador na sala de aula por professures da quinta série do ensino fundamental

como ferramenta para

proporcionar ם aprendizado

significativo nos estudantes

"Magíster en Tecnología Educativa y Medios Innovadores. Instituto Tecnológico de Monterrey - UNAB. Docente Básica Primaria,

Colegio El Pilar. Bucaramanga.

** Magíster en Administración, Universidad Santo Tomás - Docente de la Maestría en Administración de la Universidad Santo Tomás, Bucaramanga. 


\section{RESUMEN}

El objetivo de este trabajo consistió en analizar en un grupo de docentes de quinto grado de básica primaria, la inclusión de la computadora en el aula como herramienta de las Tecnologías de Información y Comunicación (TIC), que propicia el desarrollo de actividades para afrontar los grandes desafíos del aprendizaje significativo a través del proceso de enseñanza-aprendizaje. Para ello se orientó esta investigación bajo el enfoque cualitativo apoyado en el diseño etnográfico. Entre los hallazgos se encontró que los profesores en su gran mayoría utilizan estrategias que vinculan actividades programadas en el computador.

\section{ABSTRACT}

This paper aims at analyzing a group of fifth grade teachers who incorporate the use of computers in their classrooms as a tool of Information and Communication Technology (ICT), which facilitates the deveopment of class activities to definietly promote meaningful learning in the teaching-learning process. This study was made with a qualitative focus supported by an ethnographic design. Among findings, the most important one was the fact that most teachers implemented strategies which require the use of computers.

\section{RESUMÉÉ}

Le but de cette étude est d'analyser l'introduction, par un groupe d'enseignants, d'ordinateurs dans une salle de classe de cinquième année de primaire comme outil de Technologies de l'Information et de la Communication (TIC) qui favorise la mise en place d'activités novatrices relevant les défis actuels de l'enseignement et de l'apprentissage. Cette investigation a pour ce faire été réalisée dans le cadre d'une approche qualitative s'appuyant sur une conception ethnographique. Les résultats semblent mettre en évidence que les enseignants utilisent très fréquemment des stratégies qui mettent en relation les activités du programme avec l'ordinateur.

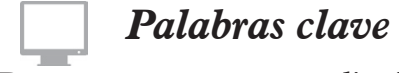

Proceso enseñanza-aprendizaje, Tecnologías de la Información y la Comunicación (TIC), la computadora, docentes.

\section{Key words}

Teaching- learning process, Information and Communication Technologies (ICT), the computer, teachers.

\section{RESUMO}

O objetivo deste trabalho consistiu em analisar em um grupo de professores da quinta série do ensino fundamental, a inclusão do computador na sala de aula como ferramenta das Tecnologias da Informação e Comunicação (TIC), que propicia o desenvolvimento de atividades para afrontar os grandes desafios da aprendizagem significativa através do processo de ensino-aprendizagem. Para isso se orientou esta pesquisa científica sob o enfoque qualitativo apoiado no desenho etnográfico. Entre as descobertas se ressalta o fato de que os professores em sua grande maioria utilizam estratégias que vinculam atividades programadas no computador. 


\section{INTRODUCCIÓN}

]

as Tecnologías de la Información y la Comunicación (TIC), se han convertido en una pieza clave en el contexto educativo y generan un suficiente número de posibilidades de gran significación en los procesos de enseñanza-aprendizaje.

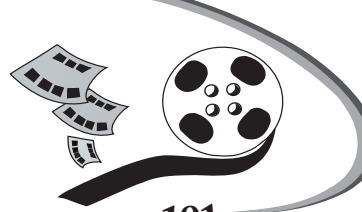

p.191
La búsqueda de caminos didácticos acordes con las nuevas posibilidades, se ha transformado en objeto de reflexión propiciando en las instituciones de educación cambios significativos en los procesos de enseñanza acordes con las exigencias de una sociedad globalizada (Cabero, 2007).

Lo anterior ha conllevado a "reevaluar los currículum tradicionales y las formas de enseñar en respuesta a los desafíos educativos producidos por la sociedad del conocimiento" (Cabero, 2007, p. 4), en donde el estudiante exige estrategias innovadoras de enseñanza de acuerdo con el ritmo tecnológico presentado en su entorno.

Es así, que autores como Marqués (2000) señala que el impacto de las TIC en la educación, puede estar dado por la importancia de los aprendizajes que las personas realizan a través de los nuevos medios de comunicación; por los cambios en los contenidos curriculares que exigen la formación integral y continua a lo largo de la vida; por la incursión de nuevas herramientas tecnológicas para la educación; por la formación permanente en competencias básicas en TIC, tanto presencial como no presencial para la actualización profesional; por la creación de nuevos entornos de enseñanzaaprendizaje que eliminan barreras de tiempo y espacio para asegurar una formación continua tanto de profesores como alumnos.

En concordancia con lo mencionado, este estudio tiene como objetivo analizar la inclusión de la computadora por parte de los docentes de quinto grado, como herramienta de las TIC que apoya el diseño de actividades que propician aprendizaje significativo en su práctica diaria.

Este artículo presenta en el apartado dos los fundamentos teóricos en donde se profundizan las temáticas que permitieron orientar la investigación y sustentar la base de este estudio. En el apartado tres se aborda lo concerniente a la metodología del proyecto, donde se explican las fases necesarias para obtener la información. En el apartado cuatro, se presentan los resultados del estudio donde se analiza la inclusión de la computadora en el aula por parte de los docentes de quinto primaria. Finalmente, en el último àpartado se presentan las conclusiones resaltando los aspectos que permitieron responder la pregunta de investigación. 


\section{FUNDAMENTOS TEÓRICOS}

\subsection{El proceso educativo fundamentado en la inclusión de las TIC}

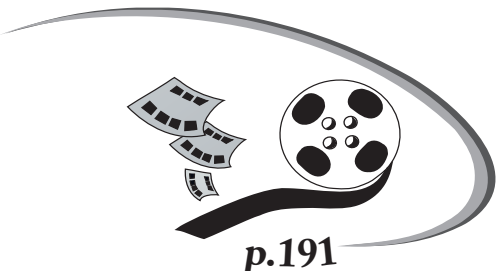

p.191
La nueva era de la información ha traído consigo el desarrollo de estrategias que buscan mejorar los procesos de enseñanzaaprendizaje en el aula. Las TIC, como instrumento para la mejora de dichos procesos, han propiciado en los docentes el diseño e implantación de nuevos planes de aula que cumplan con las exigencias que demandan la formación de los estudiantes.

No obstante, la UNESCO (2013) manifiesta que la inclusión de las TIC en el sector educativo de América Latina y el Caribe no ha reportado efectos positivos en la calidad, por lo que se deben considerar en el proceso educativo elementos como el aprendizaje significativo y la motivación del estudiante.

\subsubsection{Aprendizaje significativo}

La teoría constructivista del aprendizaje se basa en el conocimiento como una interacción entre la nueva información que se presenta y la que ya se conoce, en donde el alumno construye modelos e interpreta la información que recibe (Ausubel, Novak y Hanesian, 1999).

El aprendizaje significativo, como aspecto que destaca esta teoría, es abordado por diferentes autores. Para Ausubel "el proceso de enseñanza- aprendizaje que se produce en el propio contexto educativo, a partir de los conceptos previamente elaborados por el alumno en su vida cotidiana. Por lo tanto, desarrolla una teoría de interiorización o asimilación a través de la instrucción, de tal modo que los conceptos verdaderos se construyen a partir de los conceptos previamente formados o descubiertos por el alumno en su entorno" (Briones, 2006, pp. 156-157). Así mismo, manifiesta que deben existir tres condiciones básicas para que el agente de la enseñanza planee su desarrollo en el estudiante: la estructuración de materiales claramente jerarquizados; la identificación de la estructura psicológica del estudiante, como sus conocimientos previos y estilo de aprendizaje; y el análisis de la motivación que tiene el individuo por aprender (Ausebel, 1963; Tünnermann, 2011; Coll, 1999).

Para Piaget en su teoría constructivista del aprendizaje, señala como atributos fundamentales la organización y la adaptación como procesos fundamentales de la inteligencia, menciona que "la organización está constituida por estructuras o esquemas de conocimiento, cada una de las cuales conduce a conductas diferentes en situaciones específicas" (Briones, 2006 , p. 149) en donde el individuo construye el conocimiento a partir de una interacción con la realidad.

Por su parte, Vygotsky en su teoría sociohistórica cultural, considera el aprendizaje como una actividad mental constructivista, que implica la internalización de los significados que tienen elementos culturales en contextos de interacción social. Por lo que el alumno no es un agente pasivo sino que interactúa de manera activa con el profesor elaborando y organizando contenidos de las actividades pedagógicas.

Finalmente, la concepción constructivista del aprendizaje aporta y satisface las necesidades de un mundo cambiante y globalizado en el cual está inmersa la tecnología. Sustenta que la finalidad de la educación es promover los procesos de crecimiento personal del alumno 
en el marco de la cultura a la cual pertenece; además, propicia nuevas construcciones mentales mediante la planificación intencionada por parte del docente y la participación activa del alumno (Coll, 1999; Zubiría, 2007).

\subsubsection{Motivación escolar y sus efectos en el aprendizaje}

En el plano pedagógico, la motivación estimula la voluntad del alumno para aprender. Para Díaz et al. (2006, p.60), los profesores deben concentrarse en diseñar estrategias de apoyo, de tal forma que motiven a sus alumnos y permitan mantener un estado propicio para el aprendizaje.

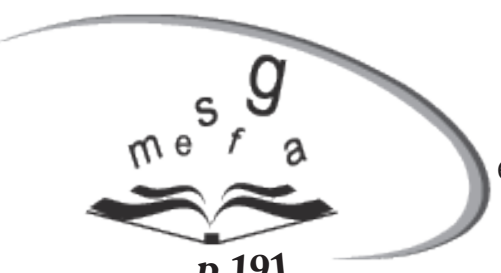

p.191
Para Cabero (2007), el nuevo rol docente debe estimular y motivar al estudiante, para que tenga una actitud favorable y pueda aprender significativamente, relacionando sus conocimientos previos con la nueva información suministrada y así modificar las estructuras cognitivas.

\subsubsection{Tecnología educativa}

En la actualidad, la tecnología forma parte de la vida cotidiana y así mismo del contexto educativo. Para Salinas (1997), el sistema educativo requiere grandes cambios propiciados por la innovación tecnológica en los nuevos ambientes de aprendizaje. Esto implica nuevas concepciones en el proceso de enseñanza y aprendizaje, caracterizadas por la relación con el saber y las nuevas prácticas de docencia adaptadas al contexto actual.

La presencia de las Tecnologías de la Información y la Comunicación (TIC), están reclamando un nuevo espacio educativo, un replanteamiento de las finalidades de la educación y la enseñanza; por lo anterior y según García y Muñoz (2008), para integrar las TIC en la enseñanza se requiere que una serie de condiciones, como la formación del profesor en su uso y posibilidades para innovar y la actitud positiva hacia los procesos generados de su implementación.

Prendes (2003), considera que las innovaciones tecnológicas ponen a disposición cambios metodológicos, cambios de actitudes en el profesorado, cambios en los objetivos, flexibilidad en el diseño de tareas y cambios organizativos que contribuyen a integrar estos medios en el aula y en la forma de dar las clases, generando nuevos entornos de aprendizaje.

Por su parte Cabero, Llorente y Román (2004), mencionan que con las nuevas tecnologías se traspasan las barreras tradicionales y se pueden crear nuevos entornos que faciliten a los usuarios su actividad formativa, independientemente del espacio y del tiempo en el cual se encuentren los actores del proceso educativo.

Sin lugar a duda, las TIC ofrecen posibilidades significativas para la creación de nuevos escenarios flexibles para interacción con recursos tecnológicos apropiados, que permitan el cambio de modelos tradicionales de comunicación.

\subsubsection{La computadora como herramienta de apoyo en el proceso de aprendizaje}

La introducción del ordenador en las aulas de educación, como señala Cabero (2000), tiene su base en la necesidad de innovación educativa porque conceptos, técnicas y modos de producir el conocimiento en la sociedad y en el aula han ido evolucionando.

Para Cabero (2007), los nuevos entornos de aprendizaje deben estar enriquecidos con materiales didácticos que lleven inmersa la 
tecnología. La nueva sociedad del conocimiento ofrece un gran potencial de herramientas y dentro de ellas se encuentra la computadora como herramienta básica para la enseñanza y el aprendizaje.

Así mismo, Ortega (2003) menciona que en los centros educativos debe integrarse el ordenador como medio facilitador en el desarrollo de actividades que van desde los usos básicos de los programas como Word, Excel, Power Point y otros, hasta la interacción a través de gran cantidad de materiales interactivos que promueven el desarrollo potencial del educando y propician aprendizaje significativo.

En términos generales, Cabero (2007) señala que la computadora en el aula ofrece entornos de trabajo en grupo, trabajo direccionado por el docente para reforzar el conocimiento o introducir a los estudiantes en un concepto concreto, el uso de juegos o programas creativos para motivar al alumno a expresarse, crear, tomar decisiones para obtener uno u otro resultado o sencillamente mejorar o reforzar habilidades, conocimientos o actitudes.

De igual manera, Medina (2003) menciona que cada día las TIC están incursionando en la educación y por ende en los centros escolares. Los alumnos de hoy ya tienen acceso a computadoras, las cuales utilizan para realizar sus actividades escolares dentro y fuera del aula de clase. Además, la computadora es una herramienta esencial de uso diario, tanto para docentes como para los educandos que facilitan el desarrollo de actividades, motivan a los educandos y se promueve la construcción de aprendizajes significativos.

\section{METODOLOGÍA}

$\mathbf{P}$ ara el estudio en particular se empleó el enfoque cualitativo, el cual ofreció grandes oportunidades de ingresar al campo para indagar, observar y describir cada uno de los acontecimientos que los individuos experimentan en su vida cotidiana (Hernández, Fernández y Baptista, 2006). De esta manera, el estudio permitió dar respuesta a la pregunta de investigación ¿Cómo los docentes emplean la computadora en el aula y propician aprendizaje significativo de los estudiantes de quinto grado de un centro educativo?

Este trabajo se apoya en la estrategia de investigación de la etnografía. San Fabián (1992) mencionado en Álvarez (2008), argumenta que la investigación etnográfica puede ser desarrollada favorablemente en contextos educativos donde los niños y jóvenes puedan ser observados en su quehacer cotidiano. Para cumplir con el proceso de recolección de información se utilizaron entrevistas semiestructuradas, grabadas en el programa Audicity y posteriormente se transcribieron en Word y Excel; así mismo, se realizó la observación directa grabada en video para obtener la mayor información posible en el estudio.

\subsection{Población}

Para la investigación se eligieron los docentes del grado quinto de primaria de un centro educativo ubicado en el Municipio del Socorro, Santander-Colombia. Para efectos del estudio se eligió por conveniencia a cinco docentes que imparten sus conocimientos en dichos grados y quienes de manera integral enseñan todas las áreas del conocimiento. 


\subsection{Instrumentos}

Para la investigación se utilizó como técnicas de recolección: las entrevistas, porque "genera la oportunidad para comprender los puntos de vista de los participantes a cerca de sus mundos tal como son descritos en sus propias palabras" (Mayan, 2001, p. 15); y la técnica de observación, puesto que debe estar atento a los "detalles, sucesos, eventos e interacciones" (Hernández, Fernández y Baptista, 2006, p.58).

\section{RESULTADOS}

I a información se recolectó a través de las entrevistas realizadas a cinco docentes y de cinco observaciones a las clases. El análisis parte del diseño de un cuadro de triple entrada como organizador de información y que se presenta como una estrategia que apoya la construcción de los instrumentos desde un objetivo muy delineado, muy definido que permite recopilar datos (Ramírez, 2008).

\subsection{Análisis de las entrevistas}

La aplicación de este instrumento, permitió identificar los medios que los docentes utilizan para integrar la computadora en el aula y propiciar el aprendizaje significativo. A continuación se presenta el consolidado de las respuestas arrojadas en la aplicación de la entrevista (Tabla 1).

Tabla 1. Consolidado de respuestas a la entrevista de docentes.

\begin{tabular}{|c|c|}
\hline Respuestas consolidadas & Respuestas consolidadas \\
\hline $\begin{array}{l}\text { 1. ¿Qué opinión tiene sobre la incorporación de } \\
\text { herramientas tecnológicas en la educación? }\end{array}$ & $\begin{array}{l}\text { - Todos los docentes las consideran como herra- } \\
\text { mientas fundamentales e importantes para el } \\
\text { aprendizaje significativo y práctico, ayuda a la } \\
\text { formación integral del educando y afianza el } \\
\text { conocimiento. }\end{array}$ \\
\hline $\begin{array}{l}\text { 2. ¿Emplea herramientas tecnológicas para } \\
\text { preparar sus clases? ¿Cuáles? }\end{array}$ & $\begin{array}{l}\text { - Tres docentes respondieron que emplean la com- } \\
\text { putadora, el Internet, el video beam, además en } \\
\text { ocasiones el TV y el DVD para reforzar algunas } \\
\text { temáticas. } \\
\text { - Dos docentes no integran ninguna herramienta } \\
\text { tecnológica. }\end{array}$ \\
\hline $\begin{array}{l}\text { 3. ¿Qué opinión tienen sobre la integración } \\
\text { de la computadora en el aula como medio de } \\
\text { aprendizaje? }\end{array}$ & $\begin{array}{l}\text { - Dos docentes emplean metodología tradicional y } \\
\text { no la consideran herramienta fundamental. } \\
\text { - Tres docentes señalan que es excelente, los alumnos } \\
\text { aprenden con mayor facilidad, permite innovar en } \\
\text { las estrategias y procesos de aprendizaje. }\end{array}$ \\
\hline $\begin{array}{l}\text { 4. ¿Qué estrategias utiliza para incorporar la } \\
\text { computadora como medio de aprendizaje? }\end{array}$ & $\begin{array}{l}\text { - Dos no la integran. } \\
\text { - Los otros tres la utilizan con actividades de intro- } \\
\text { ducción a las temáticas en resúmenes, juegos } \\
\text { didácticos, reforzar las temáticas de las diferentes } \\
\text { áreas, videos, evaluaciones. }\end{array}$ \\
\hline
\end{tabular}


Tabla 1. Consolidado de respuestas a la entrevista de docentes (Continuación)

\begin{tabular}{|c|c|}
\hline $\begin{array}{l}\text { 5. ¿Con qué frecuencia utiliza la computadora } \\
\text { como apoyo en el proceso de enseñanza- } \\
\text { aprendizaje? }\end{array}$ & $\begin{array}{l}\text { - Un docente las integra todos los días para } \\
\text { profundizar las temáticas; los otros dos docentes } \\
\text { la integran tres veces por semana. } \\
\text { - Los otros dos docentes manifiestan que la falta de } \\
\text { uso y apropiación impide integrarlas en el proceso. }\end{array}$ \\
\hline $\begin{array}{l}\text { 6. ¿Qué habilidades desarrollan los educandos } \\
\text { en el manejo de la computadora? }\end{array}$ & $\begin{array}{l}\text { - Los tres docentes aluden que los alumnos tienen } \\
\text { mucha creatividad, desarrollo mental, interacción } \\
\text { permanente, competencias básicas, concentración, } \\
\text { desarrollo motriz, motivación, colaboración, tra- } \\
\text { bajos grupales, manejo de Internet y programas } \\
\text { básicos como Word, Excel, Power Point y uso del } \\
\text { correo electrónico. } \\
\text { - Dos docentes comentaron que ninguna porque no } \\
\text { la integran. }\end{array}$ \\
\hline $\begin{array}{l}\text { 7. ¿Considera que al incorporar la computadora } \\
\text { en el aula se propicia aprendizaje significativo? } \\
\text { ¿Por qué? }\end{array}$ & $\begin{array}{l}\text { - Los tres docentes respondieron que si se propicia } \\
\text { aprendizaje significativo, porque interactúan direc- } \\
\text { tamente logrando la construcción de su propio } \\
\text { aprendizaje. El aprendizaje es más práctico, fácil y } \\
\text { duradero para la vida. } \\
\text { - Los otros dos docentes no emplean. } \\
\end{array}$ \\
\hline $\begin{array}{l}\text { 8. ¿Considera que los docentes deben recibir } \\
\text { formación sobre el uso y manejo de herra- } \\
\text { mientas tecnológicas? }\end{array}$ & $\begin{array}{l}\text { - Los cinco docentes consideran que sí deben recibir } \\
\text { formación para poder orientar correctamente en } \\
\text { el uso y manejo de herramientas tecnológicas, se } \\
\text { debe mantener actualización permanente para } \\
\text { responderalas exigencias deun mundo globalizado. } \\
\text { Aluden que un docente bien capacitado puede } \\
\text { innovar diseñar y planear apoyada con las TIC. } \\
\text { Para innovar en estrategias didácticas que generen } \\
\text { nuevos ambientes de aprendizaje y mejoren la } \\
\text { calidad educativa. }\end{array}$ \\
\hline $\begin{array}{l}\text { 9. ¿Considera que la integración de la } \\
\text { computadora como herramienta de aprendi- } \\
\text { zaje motiva a los estudiantes? }\end{array}$ & $\begin{array}{l}\text { - Los tres docentes consideran que la integración de } \\
\text { la computadora mantiene a los estudiantes concen- } \\
\text { trados, alegres, se ven con entusiasmo, se sienten } \\
\text { importantes, se sale de la monotonía. Aluden que } \\
\text { ellos se interesan más por las actividades, son más } \\
\text { creativos y colaboran con los compañeros que } \\
\text { presentan dificultad en el aprendizaje. } \\
\text { - Los otros dos docentes no integran la computadora. }\end{array}$ \\
\hline $\begin{array}{l}\text { 10. ¿Cómo es la interacción en el aula de clase } \\
\text { cuando se integra la computadora en el } \\
\text { desarrollo de actividades? }\end{array}$ & $\begin{array}{l}\text { - Los tres docentes manifiestan que mejora la disci- } \\
\text { plina, la clase se torna más participativa, dinámica, } \\
\text { fomenta la colaboración y participación de las } \\
\text { actividades, comunicación fluida entre pares e } \\
\text { interés por aprender. } \\
\text { - Los otros dos docentes no la aplican y por lo tanto } \\
\text { mencionan que no poseen experiencia. }\end{array}$ \\
\hline
\end{tabular}

Fuente. Elaboración propia de las autoras. 


\subsection{Análisis de la observación}

Los principales aspectos que se tuvieron en cuenta para la observación, se estructuraron con base en las estrategias didácticas teniendo en cuenta indicadores como: planificación, motivación y medio didáctico; para el segundo constructo que fue la tecnología, se apoyó con indicadores como: desarrollo de habilidades, manejo de la computadora e interacción, y como último constructo, se tomó el aprendizaje significativo, el cual se soportó con indicadores como: el nivel de agrado, nivel de conocimiento y construcción de conocimiento (Tabla 2).

Tabla 2. Consolidado observación directa no participativa a docentes

\begin{tabular}{|c|c|c|}
\hline Categoría & Indicador & Aspectos observados \\
\hline \multirow{3}{*}{$\begin{array}{l}\text { Estrategias } \\
\text { didácticas }\end{array}$} & Planificación & $\begin{array}{l}\text { - Dos docentes planificaron sus clases de forma tradicional em-pleando } \\
\text { solo textos y guías manuales. } \\
\text { - Tres docentes, planificaron de manera flexible sus estrategias, para dar } \\
\text { participación a sus estudiantes, tomando el tiempo requerido para cada } \\
\text { una de las actividades programadas. Emplearon guías específicas que } \\
\text { contenían objetivos, justificación y detalles de las actividades. }\end{array}$ \\
\hline & Motivación & $\begin{array}{l}\text { - En los dos docentes que no emplean la tecnología, se observó poca } \\
\text { motivación de los estudiantes en el desarrollo de sus clases las cuales } \\
\text { fueron monótonas. } \\
\text { - En los docentes que integran las TIC se notó que logran captar la } \\
\text { atención de sus alumnos, emplean videos y diapositivas relacionados } \\
\text { con las temáticas, con lo pudieron mantener concentrados y atentos a } \\
\text { sus estudiantes. Además, se interesaron de una manera más dinámica } \\
\text { en dichas temáticas. }\end{array}$ \\
\hline & $\begin{array}{c}\text { Medio } \\
\text { didáctico }\end{array}$ & $\begin{array}{l}\text { - En la investigación se presentan dos docentes que no emplean la } \\
\text { tecnología. } \\
\text { - Los otros tres docentes emplearon el computador, el video beam y un } \\
\text { Software educativo, fotocopias y programa de Word, estos docentes } \\
\text { emplearon juegos didácticos e interactivos como estrategia para } \\
\text { profundizar las temáticas. }\end{array}$ \\
\hline \multirow{3}{*}{$\begin{array}{c}\text { Tecnología } \\
\text { (Manejo de la } \\
\text { computadora) }\end{array}$} & $\begin{array}{l}\text { Desarrollo de } \\
\text { habilidades }\end{array}$ & $\begin{array}{l}\text { - Los estudiantes adquieren destrezas en el manejo de formatos con el } \\
\text { programa Word. El docente promueve actividades de investigación, con } \\
\text { programas pedagógicos (educativos) como Encarta. } \\
\text { - El docente se encarga de que los estudiantes dirijan la atención, mejoren } \\
\text { la retención de las actividades y promuevan el aprendizaje a través de la } \\
\text { realización de una serie de actividades. }\end{array}$ \\
\hline & $\begin{array}{l}\text { Manejo de la } \\
\text { computadora }\end{array}$ & $\begin{array}{l}\text { Los estudiantes poseen un buen manejo de la computadora y realizan } \\
\text { el ejercicio con gran facilidad. Tanto los docentes como los estudiantes } \\
\text { tienen un manejo básico de esta y utilizan Word donde es utilizada como } \\
\text { una guía mediante el uso de programas educativos matemáticos. Realizan } \\
\text { diagramas con el programa de Paint. }\end{array}$ \\
\hline & Interacción & $\begin{array}{l}\text { - Se observó una interacción adecuada al tema, donde el profesor es guía } \\
\text { y orientador de la clase. También se identificó en los estudiantes una } \\
\text { constante interacción; con el docente con el fin de recibir orientación, } \\
\text { así los estudiantes analizan, comparten sus inquietudes, al ser un grupo } \\
\text { pequeño de alumnos la interacción es más oportuna y continua. } \\
\text { - La interacción entre docente y estudiantes es adecuada, se evidenció } \\
\text { trabajo colaborativo. }\end{array}$ \\
\hline
\end{tabular}


Tabla 2. Consolidado observación directa no participativa a docentes (Continuación)

\begin{tabular}{|c|c|c|c|}
\hline & $\begin{array}{c}\text { Nivel de } \\
\text { agrado }\end{array}$ & $\begin{array}{c}\text { - Se observó excelente motivación hacia las temáticas, se notó gran interés } \\
\text { en las actividades que desarrollaron los estudiantes, estuvieron atentos, } \\
\text { disciplinados, se identificó que las actividades fueron más practicas e } \\
\text { interactivas y se vieron involucrados el proceso de aprendizaje. }\end{array}$ \\
\hline \multirow{2}{*}{$\begin{array}{c}\text { Aprendizaje } \\
\text { significativo }\end{array}$} & $\begin{array}{c}\text { Nivel de } \\
\text { conocimiento observó en los estudiantes una gran capacidad de crear su propio } \\
\text { conocimiento a través de cada actividad, indagando, compartiendo y } \\
\text { experimentando aprendizajes. Compartirla y disfrutarla; un modelo de } \\
\text { clase que sea creativo, innovador y participativo. Se observaron buenas } \\
\text { actitudes en los estudiantes hacia el conocimiento y su interés por } \\
\text { aprender. }\end{array}$ \\
\cline { 2 - 4 } & $\begin{array}{c}\text { Construcción } \\
\text { de } \\
\text { conocimiento }\end{array}$ & $\begin{array}{l}\text { La construcción de conocimiento se realizó de una manera creativa, } \\
\text { innovadora y participativa. En estas clases se observó que se aprovecharon } \\
\text { los recursos humanos y la cooperación de todos. También los estudiantes } \\
\text { adquirieron destrezas y participaron a través de las diferentes actividades } \\
\text { en la construcción de un aprendizaje más significativo y duradero. Se } \\
\text { notó en los estudiantes el desarrollo del pensamiento lógico matemático. }\end{array}$ \\
\hline
\end{tabular}

Fuente. Elaboración propia de las autoras.

Los resultados reportaron que los docentes que emplean el computador como herramienta de apoyo en su actividad pedagógica, programan sus actividades de manera flexible para motivar a sus estudiantes, como sujetos activos en el proceso de enseñanza-aprendizaje.
Por otra parte, la interpretación de los resultados se confrontó con el marco teórico de la investigación y se realizó de acuerdo con los indicadores establecidos como son: planificación, motivación y medio didáctico (Tabla 3).

Tabla 3. Uso de la computadora

\begin{tabular}{|c|c|c|}
\hline Indicador & Resultados & Fundamentos teóricos \\
\hline $\begin{array}{l}\text { Desarrollo de } \\
\text { habilidades }\end{array}$ & $\begin{array}{l}\text { Los tres docentes que hacen uso de las TIC, } \\
\text { manifestaron que, integrando la compu- } \\
\text { tadora en el aula, los alumnos tienen mucha } \\
\text { creatividad, desarrollo mental, interacción } \\
\text { permanente, concentración, motivación, } \\
\text { entre otros. Lo anterior se corroboró por } \\
\text { medio de la observación de las clases, en } \\
\text { donde los estudiantes demostraron tener } \\
\text { destrezas en el desarrollo de diferentes } \\
\text { actividades dirigidas por los docentes. }\end{array}$ & $\begin{array}{l}\text { Estos hallazgos se sustentan con lo } \\
\text { mencionado por Gily García (1995), quienes } \\
\text { señalan que los ordenadores ofrecen la } \\
\text { posibilidad de que los alumnos puedan } \\
\text { alcanzar destrezas y habilidades, construyan } \\
\text { aprendizajes significativos, y sean más } \\
\text { autónomos en su proceso de aprendizaje. }\end{array}$ \\
\hline $\begin{array}{l}\text { Manejo de la } \\
\text { computadora }\end{array}$ & $\begin{array}{l}\text { Tres docentes mencionaron que integran } \\
\text { la computadora en actividades de intro- } \\
\text { ducción y refuerzo de las temáticas en las } \\
\text { diferentes áreas en: resúmenes, juegos } \\
\text { didácticos, videos y evaluaciones. Alu- } \\
\text { dieron que los estudiantes tienen buen } \\
\text { dominio de la misma en el desarrollo de } \\
\text { diversas actividades que se programan en } \\
\text { el aula de clase. }\end{array}$ & $\begin{array}{l}\text { Este indicador se respalda con lo señalado } \\
\text { por Cabero (2000), quien menciona que } \\
\text { el ordenador debe integrarse en las aulas } \\
\text { desde temprana edad empezando por la } \\
\text { educación infantil, puesto que tiene su base } \\
\text { en la necesidad de innovación educativa } \\
\text { y esto conlleva a tener nuevos conceptos, } \\
\text { nuevas técnicas y modos de producir el } \\
\text { conocimiento. }\end{array}$ \\
\hline
\end{tabular}


Tabla 3. Uso de la computadora (Continuación)

\begin{tabular}{|c|l|l|}
\hline \multirow{3}{*}{ Interacción } & Los tres docentes manifestaron que & Según Ortega (2013), la comunicación en \\
integrando la computadora en el aula se & las aulas de clase debe ser bidireccional \\
obtienen mejores resultados deaprendizaje, & integrando diversidad de medios tecno- \\
entre ellos: mejora la disciplina, la clase & lógicos entre ellos el ordenador y facilitando \\
se torna más participativa, dinámica, se & el desarrollo de actividades con programas \\
fomenta la colaboración y participación en & básicos como Word, Excely Paint. \\
& las actividades, comunicación fluida entre \\
pares e interés por aprender. & \\
\hline
\end{tabular}

\section{Fuente. Elaboración propia de las autoras.}

Según lo hallado anteriormente, se resume la inclusión de la computadora como herramienta que propicia ambientes innovadores de enseñanza y aprendizaje. Para triangular la información se toman los indicadores de desarrollo de habilidades, manejo de la computadora e interacción. Se observa que los estudiantes responden con actitudes de motivación e interés a estas iniciativas del docente (Tabla 4).

Tabla 4. Aprendizaje significativo

\begin{tabular}{|c|c|c|}
\hline Indicador & Resultados & Fundamentos teóricos \\
\hline $\begin{array}{l}\text { Nivel de } \\
\text { agrado }\end{array}$ & $\begin{array}{l}\text { Con la utilización de la computadora como } \\
\text { apoyo en las clases, se logra mantener el } \\
\text { interés de los educandos por las temáticas } \\
\text { además del desarrollo de las actividades con } \\
\text { más entusiasmo y creatividad. Esto se pudo } \\
\text { justificar en la observación donde se apreció } \\
\text { la motivación hacia las temáticas, el interés } \\
\text { en el desarrollo de las actividades y una mejor } \\
\text { disciplina. }\end{array}$ & $\begin{array}{l}\text { Díaz y Hernández (2006), señalan que el } \\
\text { docente juega un papel fundamental, es } \\
\text { quien crea situaciones o ambientes de } \\
\text { aprendizaje en donde debe involucrar a } \\
\text { sus alumnos para que fluya un lenguaje } \\
\text { de interacción permanente y el aprendizaje } \\
\text { significativo. }\end{array}$ \\
\hline $\begin{array}{c}\text { Nivel de } \\
\text { conocimiento }\end{array}$ & $\begin{array}{l}\text { Los hallazgos reportaron que la integración de } \\
\text { la computadora en su quehacer pedagógico, } \\
\text { propicia un mejor nivel de conocimiento, } \\
\text { puesto que los ambientes de aprendizaje son } \\
\text { más prácticos e innovadores. Los estudiantes } \\
\text { que realizan las actividades, son creativos y } \\
\text { trabajan colaborativamente. }\end{array}$ & $\begin{array}{l}\text { Coll (2007) señala que la escuela debe } \\
\text { formar alumnos que aprendan y desarrollen } \\
\text { el conocimiento como proceso constructivo, } \\
\text { donde los sujetos poseen ideas previas a } \\
\text { partir de las cuales inician la construcción de } \\
\text { nuevos conocimientos. }\end{array}$ \\
\hline $\begin{array}{c}\text { Construcción } \\
\text { de } \\
\text { conocimiento }\end{array}$ & $\begin{array}{l}\text { La integración de la computadora como apoyo } \\
\text { en su práctica pedagógica propicia en los } \\
\text { estudiantes la construcción de conocimiento } \\
\text { de manera creativa, innovadora y participativa. } \\
\text { Dentro de las actividades propuestas, se } \\
\text { percibió en los estudiantes el desarrollo del } \\
\text { pensamiento lógico matemático y habilidades } \\
\text { comunicativas. }\end{array}$ & $\begin{array}{l}\text { Ballester (2002) señala que para que se dé } \\
\text { un aprendizaje auténtico y a largo plazo, es } \\
\text { necesario conectar la estrategia didáctica del } \\
\text { profesorado con las ideas previas del alumno } \\
\text { para que se logre un verdadero aprendizaje } \\
\text { significativo. }\end{array}$ \\
\hline
\end{tabular}

Fuente. Elaboración propia de las autoras.

En la información anterior, se presentan aspectos concernientes a los indicadores de agrado, nivel de conocimiento y construcción de conocimiento. Desde los resultados obtenidos, se determina que los estudiantes, por medio del uso de la computadora, apropian con mayor facilidad el nuevo conocimiento, puesto que los ambientes de aprendizaje son más prácticos e innovadores lo cual despierta el interés de aprender en el estudiante. 


\section{CONCLUSIONES}

$\mathbf{E}$ n la investigación se identificó que tres de los cinco docentes emplean estrategias de enseñanza-aprendizaje que incorporan la computadora como herramienta. Los docentes están de acuerdo con el uso de herramientas tecnológicas para propiciar el aprendizaje significativo; en este caso manifiestan que la computadora y otros elementos permiten reforzar algunas temáticas impartidas en clase, innovar en las estrategias del proceso de enseñanza-aprendizaje y aumentar el nivel de motivación de los estudiantes a aprender. No obstante, aún se presenta la resistencia de algunos profesores para ser incluida.

La elaboración de resúmenes, el desarrollo de juegos didácticos, el fortalecimiento de temáticas en diferentes áreas, la proyección de videos y la realización de evaluaciones, son algunas de las actividades adoptadas por los docentes para integrar el uso de la computadora en el aula de clase.
Los profesores argumentan que los estudiantes, al utilizar la computadora, desarrollan aspectos como la creatividad, el desarrollo mental, la interacción permanente, las competencias básicas, la concentración, el desarrollo motriz, la motivación, la colaboración, el trabajo en equipo, el manejo de Internet, los programas básicos como Word, Excel, PowerPoint y el uso del correo electrónico.

Con respecto al aprendizaje significativo, los docentes argumentan que el uso de la computadora promueve la participación del estudiante en el proceso de apropiación del conocimiento, pues lo realizan de manera creativa, innovadora y participativa, siendo más práctico, fácil y duradero para la vida.

De acuerdo con las estrategias que utilizan los docentes para la inclusión de la computadora en el aula, se determina que la planificación es flexible ajustándose a la participación de los estudiantes. La motivación en el aula es mucho mayor cuando se utiliza la computadora, existe mayor atención, interés y concentración a las orientaciones y actividades propuestas por el docente.

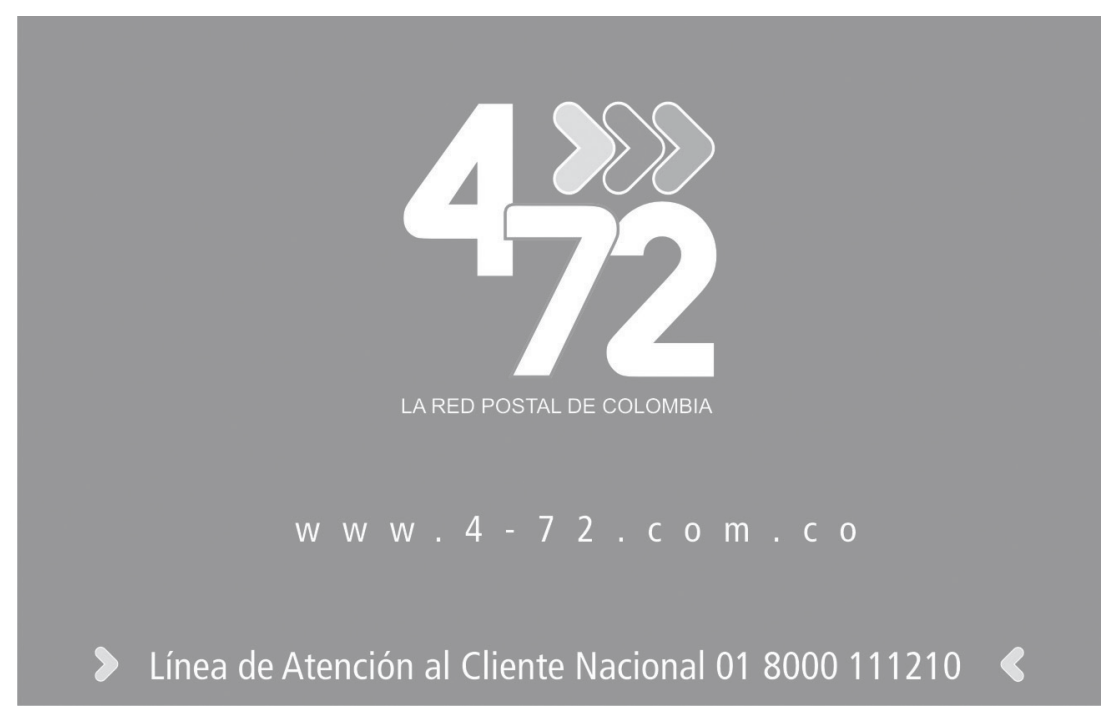




\section{REFERENCIAS BIBLIOGRÁFICAS}

Alvarez, C. (2008). La etnográfia como modelo de investigación en educación. Gazeta de Antropología.

Ausubel, D., Novak, J. y Hanesin, H. (1999). Psicología Educativa. Un punto de vista cognoscitivo. México: Trillas. 2a. Edición.

Ballester, A. (2002). El aprendizaje significativo en la práctica. Recuperado de https://docs.google. com/gview?url=http://www.aprendizajesignificativo.es/mats/Elaprendizaje_significativo_en_la_ practica.pdf\&chrome=true

Briones, G. (2006). Teorías de las ciencias sociales y de la educación: Epistemología. Capítulo 11: Teorías de la educación. Trillas. ISSN/ISBN: 978-968-24-7664-8.147-163.

Cabero, J. (2000). Las Nuevas Tecnologías de la Información y de la Comunicación: aportaciones a la enseñanza. Madrid. Ed. Síntesis.

Cabero, J, Llorente, M. C. y Román, P. (2004). Las herramientas de comunicación en el aprendizaje mezclado. (s.c) (s.f).

Cabero, J (2007). Nuevas Tecnologías aplicadas a la educación. España: Mac Graw Hill.

Coll, C. (1999). Algunos desafíos de la educación básica en el umbral del nuevo milenio. Perfiles Educativos, enero-junio, 83/84. Universidad Nacional Autónoma de México. México D. F. Red de Revistas Científicas de América Latina y el Caribe, España y Portugal Sistema de Información Científica Redalyc. Recuperado de: http://redalyc.uaemex.mx/src/inicio/ArtPdfRed. jsp?iCve $=13208402 \& i$ i veNum $=197$

Coll, C. (2007). El constructivismo en el aula. recuperado. de: http://books.google.com.co/books?id=B zOef9UIDb4C\&printsec=frontcover\&dq=coll\&hl=es\&sa=X\&ei=fX9FT46gOcL8ggfJzazHBA\&ved= 0CC8Q6AEwAA\#v=onepage\&q=coll\&f=falseEl constructivismo en el aula Escrito por César Coll

Corredor, M., Pérez, M., Arbeláez, R. (2008). Estrategias de enseñanza y aprendizaje. Bucaramanga: Cedeuis.

De Zubiría, M. (2007). Enfoques pedagógicos y didácticas contemporáneas. Colombia: Fundación Internacional de Pedagogía conceptual.

Díaz, F. y Hernández, G. (2003). Cognición situada y estrategias para el aprendizaje significativo. Revista Electrónica de Investigación Educativa, 5 (2). Recuperado de: http://redie.ens.uabc.mx/vol5no2/ contenido-arceo.html.

Díaz, B. y Hernández, G. (2006). Estrategias docentes para un aprendizaje significativo: una interpretación constructivista. México: McGraw-Hill.

Gil, G. y García, A. (1995). El ordenador como recurso didáctico. Madrid. Ed UNED

García, A. y Muñoz, V. (2008). Investigación y Tecnologías de la Información y Comunicación al servicio de la innovación educativa. Salamanca, España: Ediciones Universal. Recuperado de: http:// books.google.com.co/books?id=kjeplOHXo58C \&printsec=frontcover\&hl=es\&source=gbs_ge_su mmary_r\&cad $=0 \# v=$ onepage $\& q \& f=f a l s e$ 
González, V. (2001). Estrategias de enseñanza y aprendizaje. Editorial Pax México.

Hernández R., Fernández, C. \& Baptista, P. (2006). Metodología de la Investigación. Distrito Federal, México: Mac Graw Hill, 4 a edición.

Hernández R., Fernández, C. \& Baptista, P. (2010). Metodología de la Investigación. Distrito Federal, México: Mac Graw Hill, $5^{a}$ edición.

Marqués, P. (2008). Las competencias digitales en los docentes. Recuperado de: http://peremarques. pangea.org/competenciasdigitales.htm

Mayan, M. (2001). Una introducción a los métodos cualitativos: módulo de entrenamiento para estudiantes y profesionales. Recuperado de: http://tecnoeduka.110mb.com/documentos/ investiga/libros/mayan\%20-\%20intcuali.pdf.

Marzano, R y Pickering, J. (2005). Dimensiones del aprendizaje. Manual para el maestro. México:Ed. ITESO, pp.303-339. Archivo: Marzano 2001.pdf

Medina, M. (2003). El uso del ordenador en educación infantil: ¿Un desafío o una realidad? Recuperado de: http://edutec.rediris.es/Revelec2/Revelec17/tesouro_16a.htm.

Monereo, C. Castello, M. Clariana, M. Palma, M. y Pérez, M. (2007). Estrategias de enseñanza y aprendizaje. Formación del profesorado y aplicación en la escuela. Barcelona: Editorial Graó. Recuperado de: http://books.google.es/books?id=wV6a50yWP74C\&printsec=frontcover\&hl=es \&source=gbs_ge_summary_r\&cad=0\#v=onepage\&q\&f=false

Ortega, J. (2003). La alfabetización digital. Perspectivas creativas y éticas. En M.V. Aguiar y J. I Farray (Coordr.). Sociedad de la Información y Cultura Mediática. La Coruña: Netbiblo, pp. 91.118.

Prendes, M., P. y otros (2003). La enseñanza ante el desarrollo tecnológico del siglo XXI. Recuperado de: http://tecnologiaedu.us.es/tecnoedu/index.php?option=com_content\&view=article\&id=24:d ocme\&catid=13:articulos.

Ramírez, M. S. (2008). Triangulación e instrumentos para análisis de datos. Recuperado de: http:// sesionvod.itesm.mx/acmcontent/b98fca5b-7cb6-4947-b8de-41ac3d3cdb9c/Unspecified_ EGE_2008-06-19_05-29-p.m..htm

Salinas, J. (1997). Nuevos ambientes de aprendizaje para una sociedad de información. Revista Pensamiento Educativo. PUC Chile. Recuperado de: www.uu.es/depart/gte/ambientes.html

Maroto, S. (1992). Evaluación etnográfica de la educación. Perspectivas de la evaluación del sistema educativo. Universidad de Oviedo, pp. 13-53

Tünnermann Bernheim, C. (2011). El constructivismo y el aprendizaje de los estudiantes. Universidades, (48), enero-marzo, pp. 21-32. ISSN: 0041-8935

UNESCO (2008). Estándares UNESCO de competencia en TIC para docentes. Eduteca. Tecnología de Información y la Comunicación para la Enseñanza Básica y Media. Recuperado de: http://www. eduteka.org/modulos/11/342/868/1

UNESCO (2013). Enfoques estratégicos sobres las TICS en educación de América Latina y el Caribe. Recuperado de: http://www.unesco.org/new/fileadmin/MULTIMEDIA/FIELD/Santiago/images/ ticsesp.pdf 